DOI 10. 18307/2017. 0323

(C) 2017 by Journal of Lake Sciences

\title{
高山湖泊吉仁错枝角类古生态变化及其对气候环境的响应”
}

\author{
孔令阳,羊向东**, 王 倩 \\ (中国科学院南京地理与湖泊研究所湖泊与环境国家重点实验室,南京 210008)
}

\begin{abstract}
摘 要: 高山湖泊吉仁错位于川西高原树线以上, 受现代冰川融水补给影响. 通过吉仁错沉积钻孔 ${ }^{210} \mathrm{~Pb} /{ }^{137} \mathrm{Cs}$ 定年和沉 积枝角类和多环境代用指标分析, 结合主成分分析和几余分析等方法, 高分辨率重建了近 $200 \mathrm{a}$ 来吉仁错枝角类组合和 环境变化的过程. 结果表明,尽管过去 $200 \mathrm{a}$ 来吉仁错枝角类组合一直以沿岸种为主,但仍经历了 3 个明显的变化阶段. $1850 \mathrm{AD}$ 后, 枝角类组合中 Alonella nana、Chydorus sphaericus 和 Pleuroxus sp. 的增加、以及 Alona rustica 和 Alona guttata 丰度 的下降指示了水温和水体 $\mathrm{pH}$ 值的上升. $1900 \mathrm{AD}$ 后枝角类通量的增高并不同步于组合的变化, 指示了湖泊营养开始增 加, 这种不一致的变化分别代表了大气氮沉降和区域气候变暖的影响结果. 多指标综合分析得出, 过去 $150 \mathrm{a}$ 来, 气候变 暖和大气污染沉降通过直接和间接作用 (流域冰川融水过程和植被土壤过程), 较深刻地影响了湖泊物理过程 (水温升高 和无冰期加长) 、营养过程 (氮、磷营养升高) 和酸碱平衡过程 (碱性增强), 改变了湖泊生物的生长季节, 并通过促进藻类 发育, 最终引起了湖泊枝角类群落组合的变化和生物量的增加. $1945 \mathrm{AD}$ 前后吉仁错枝角类与藻类群落结构和湖泊环境 的同步变化,响应于持续增温背景下多环境过程的相互作用.
\end{abstract}

关键词: 沉积枝角类;古生态;气候变化;大气沉降;冰融水;高山湖泊;吉仁错

\section{Paleoecological change of cladoceran and the response to climatic environmental change from an alpine lake-Jiren Co}

\author{
KONG Lingyang, YANG Xiangdong ** \& WANG Qian \\ (State Key Laboratory of Lake Science and Environment, Nanjing Institute of Geography and Limnology, Chinese Academy of \\ Sciences, Nanjing 210008,P.R.China)
}

\begin{abstract}
Jiren Co is a small oligotrophic lake in West Sichuan plateau, located above the present tree-line and influenced by modern glacier meltwater input. Based on ${ }^{210} \mathrm{~Pb} /{ }^{137} \mathrm{Cs}$ dating and multi-proxies (i.e., cladoceran, diatom and geochemical) from Jiren Co sediment core and using principal component analysis and redundancy analysis, this study analyzed the history of cladoceran assemblage change during the past 200 years, and reconstructed the environmental change of Jiren Co. Although cladoceran remains in Jiren Co was dominated by littoral species with significant changes over time, the core assemblages can divided into three distinct zones. After $1850 \mathrm{AD}$, the increase in abundance of Alonella nana, Chydorus sphaericus and Pleuroxus sp. and the decrease in Alona rustica and Alona guttata all indicate rising temperature and pH. After 1900 AD, cladoceran flux increased earlier than the change in abundance, which indicates the beginning of lake nutrition increases. This inconformity reflects the influence of climate warming and atmospheric nitrogen deposition, respectively. Multi-index analysis suggests that, over the past 150 years, climate warming and atmospheric deposition in the directly and indirectly effects (ice melt, vegetation and soil development) have affected the physical processes on the water temperature rise and prolonged ice-free period, nutrition processes on nitrogen and phosphorus increased, acid-base equilibrium processes on the increased alkalin. It changed the growing season and promoted the growth of algae, and finally affected on the assemblage and production of cladoceran. The synchronization change of cladoceran assemblage, algae and geochemistry proxies indicates a response of alpine lake to multi-environmental forcing under the background of continuous climate warming.
\end{abstract}

Keywords: Cladoceran remains; paleoecology; climate change; atmospheric deposition; glacial meltwater; alpine lake; Jiren Co

* 国家重点研发计划项目(2016YFA0600500) 和国家自然科学基金项目 (41272379) 联合资助. 2016-08-18 收稿; 2016-09-26 收修改稿. 孔令阳(1985 ), 男,博士;E-mail : konglingyang20504@ 163.com.

** 通信作者;E-mail:xdyang@ niglas.ac.cn. 
增温和持续加强的人类活动干扰是过去一个世纪全球变化的主要特征 ${ }^{[1]}$, 其生态效应是备受关注的热 点议题. 高山地区对气候变化响应非常敏感, 增温引起的生态效应较其他地区可能更为显著 ${ }^{[2]}$. 尽管高山地 区通常远离人类活动的直接影响, 但由化石燃料燃烧和化肥施用等造成的全球或区域性大气污染 ${ }^{[3-4]}$, 通过 湿沉降, 也逐渐成为高海拔地区不断增强的环境压力 ${ }^{[5-6]}$. 最新的研究发现, 我国西南高山地区大气活性氮 沉降速率已经接近临界载荷值 ${ }^{[7]}$, 高山湖泊普遍出现营养盐积蓄等问题 ${ }^{[6-8]}$. 通常, 气候变化和大气污染沉 降通过影响高山湖泊理化特征, 进而引起湖泊生物群落结构的改变 ${ }^{[9-10]}$. 然而, 复杂的地质背景、地形地貌、 垂直植被和土壤分布等湖泊流域特征, 使得高山湖泊生态系统对外部驱动力的响应方式变得更为复杂 ${ }^{[11]}$, 而如何分辨多环境胁迫因子对生态过程影响的相对重要性, 揭示气候变化和人类活动相互作用下高山湖泊 生态系统的响应机理,达到预测未来的目的, 已成为古湖沼学领域关注的一个重要内容.

位于我国青藏高原东南缘的西南横断山脉地区, 是全球生物多样性最丰富的地区 ${ }^{[12]}$, 区域内分布着众 多的小型高山湖泊,大多位于树线附近, 属于冰川活动形成的遗迹湖. 在更高海拔的山体顶部, 仍有一些现 代冰川发育, 规模通常较小, 这些高山湖泊明显受冰川融水补给. 目前在该区只有少数的高山湖泊的古湖沼 研究工作 ${ }^{[6,13-16]}$. 前期的研究主要利用沉积硅藻记录, 着重关注过去百年来湖泊藻类生态变化对增温的响 应 ${ }^{[13-15]}$, 近期开始利用硅藻、色素和枝角类等多古生态指标, 揭示气候变化与大气污染沉降对生态的影 响 ${ }^{[6,16]}$, 并初步探讨了多因子相互作用对生态过程的影响机理. 然而, 这些研究主要局限于树线附近的森林 湖泊和无冰川补给的湖泊类型, 对更高海拔的受冰川补给湖泊的古生态研究目前尚缺, 这也限制了研究区 不同类型高山湖泊的生态变化对比以及区域规律性认识的获得.

枝角类是湖泊食物链上的重要组成部分, 具有生命周期相对较短且响应快速的特点. 并且由于其分类 清晰、化石能较好地保存, 可以根据沉积物中枝角类微化石的种类组成、形态和数量等信息推断沉积时的水 体环境乃至影响水体的气候环境 ${ }^{[17-20]}$. 本研究选择川西高原一个受冰川融水补给的高山湖泊一一吉仁错作 为研究对象, 以沉积枝角类为主要的代用指标, 并结合硅藻和地球化学指标, 重建近 $200 \mathrm{a}$ 来吉仁错生态变 化过程, 结合区域温度变化和氮沉降数据, 分析气候变化以及大气沉降对高山湖泊的影响, 探讨冰川融水补 给湖泊生态变化的机制, 为预测未来全球变化情景下湖泊生态系统的可能变化趋势提供重要依据.

\section{1 研究区概况}

吉仁错位于甘孜藏族自治州雅江县(图 1), 海拔高度 $4480 \mathrm{~m}$ a.s.l.. 该湖位于树线以上,属于冰蚀湖. 流 域呈盆地地形, 湖泊东、北、西三面为陡峭的山体, 多为裸露的岩石, 山体顶部有少量山岳冰川覆盖. 除降水 补给外, 湖泊还接纳流域冰川和积雪融水补给, 唯一出水口位于东面. 流域面积 $4.71 \mathrm{~km}^{2}$, 湖泊面积 0.12 $\mathrm{km}^{2}$, 最大深度 $28.5 \mathrm{~m}$, 流域和湖泊面积之比约为 40 . 该湖水体清澈, 呈弱碱性, 营养水平低 (表 1). 流域植被 主要集中在湖泊西岸和西北岸, 为矮化高山杜鹃 (Rhododendron lapponicum) 灌丛覆盖. 该湖流域没有直接的 人类活动迹象.

该湖所在区域主要受西南季风和南支西风的影响. 冬半年 (11 月至次年 4 月)气候偏冷干;夏半年 (510 月) 季风降水充沛. 据吉仁错附近气象站点 (理塘: 海拔 $3949 \mathrm{~m}$, 距吉仁错 $39 \mathrm{~km}$ ) 近 55 年来的温度和降水 气象数据 (数据来源于国家气候中心 http://ncc.cma.gov.cn/cn/) 显示, $6-8$ 月温度最高, 12 月至次年 2 月 温度最低. 理塘气象站点年均降雨量为 $730 \mathrm{~mm}$,大多集中在 5-9 月,占全年总降雨量的 $89 \%$.

\section{2 材料与方法}

\section{1 样品采集}

2014 年 5 月利用 Hon-Kajak 大口径 (直径 $9 \mathrm{~cm}$ ) 重力采样器在吉仁错湖心 $28 \mathrm{~m}$ 深处 (图 1) 采集长度为 $38 \mathrm{~cm}$ 的沉积柱. 现场对钻孔沉积物按 $0.5 \mathrm{~cm}$ 间隔进行分样, 样品保存在自封袋中放于 $4^{\circ} \mathrm{C}$ 的冰箱中冷藏以 备分析. 现场对湖泊水深、水体透明度、溶解氧 (DO) 浓度、 $\mathrm{pH}$ 值、电导率、浊度和叶绿素 a 浓度指标进行测 量, 并采集表层水样用于湖水总磷 $(\mathrm{TP})$ 和总氮 $(\mathrm{TN})$ 浓度分析. 测试的水环境数据见表 1 .

\section{2 实验室分析}

实验室内, 对钻孔沉积柱进行了 ${ }^{210} \mathrm{~Pb}$ 和 ${ }^{137} \mathrm{Cs}$ 年代测试, 分析的沉积物指标包括总有机碳 (TOC) 含量、 


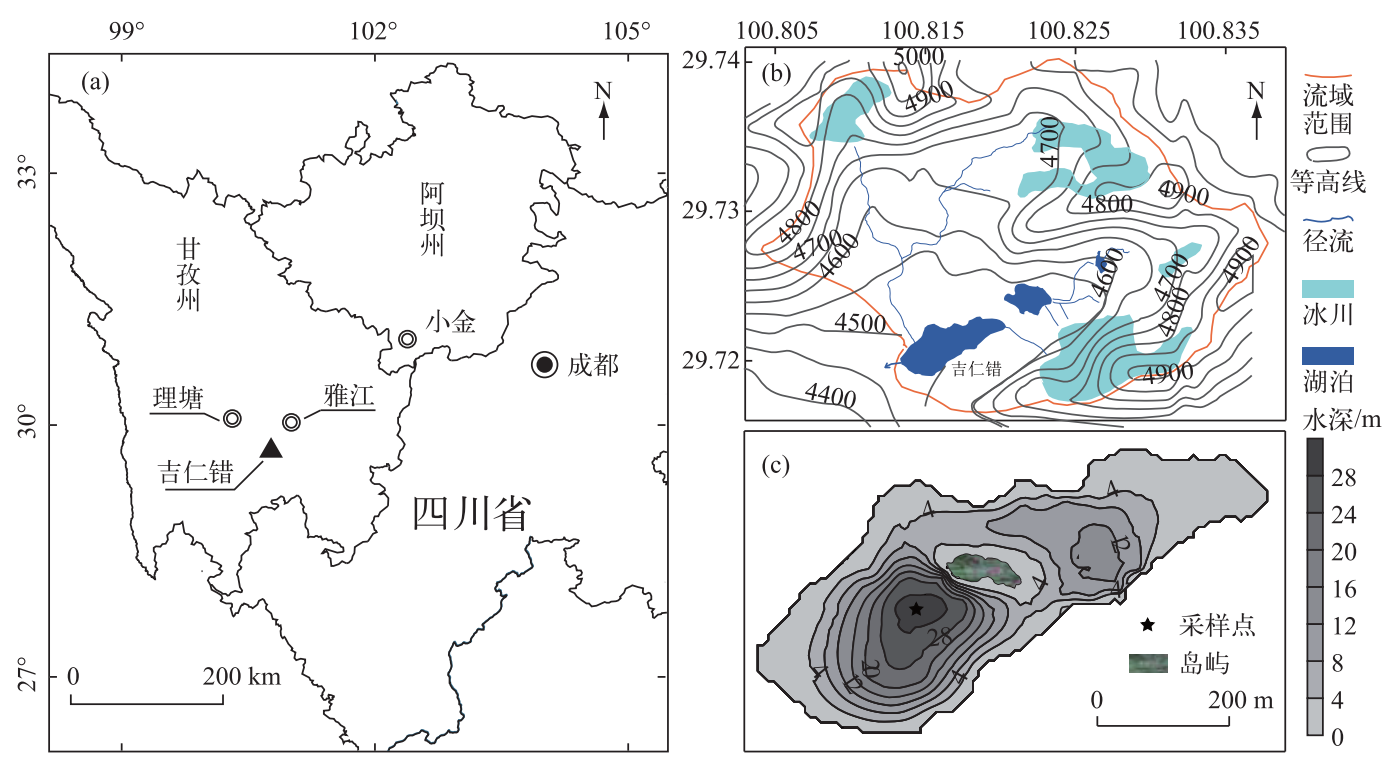

图 1 吉仁错地理位置( a)、流域概况 (b)、采样位点及等深图 (c)

Fig.1 Location of Jiren Co (a), the catchment (b), lake morphometry and the location of sampling sediment core (c) of Jiren Co

表 1 吉仁错基本环境特征

Tab.1 Environmental characteristics of Jiren Co

\begin{tabular}{cccccccccc}
\hline $\begin{array}{c}\text { 水深/ } \\
\mathrm{m}\end{array}$ & $\begin{array}{c}\text { 透明度/ } \\
\mathrm{m}\end{array}$ & $\begin{array}{c}\text { 溶解氧/ } \\
\%\end{array}$ & $\begin{array}{c}\text { 盐度/ } \\
\%\end{array}$ & $\mathrm{pH}$ & $\begin{array}{c}\text { 电导率/ } \\
(\mathrm{S} / \mathrm{m})\end{array}$ & $\begin{array}{c}\text { 浊度/ } \\
(\mu \mathrm{g} / \mathrm{L})\end{array}$ & $\begin{array}{c}\text { 叶绿素 } \mathrm{a} / \\
(\mathrm{mg} / \mathrm{L})\end{array}$ & $\begin{array}{c}\mathrm{TN} / \\
(\mathrm{mg} / \mathrm{L})\end{array}$ & $\begin{array}{c}\mathrm{TP} / \\
(\mathrm{mg} / \mathrm{L})\end{array}$ \\
\hline 28 & 8.5 & 6.3 & 0.024 & 7.4 & 0.046 & 5.9 & $<0.001$ & 0.62 & 0.083 \\
\hline
\end{tabular}

$\mathrm{TN}$ 含量、氮同位素 $\left(\delta^{15} \mathrm{~N}\right)$ 、金属和重金属元素含量、硅藻和枝角类含量. 所有指标均在中国科学院南京地理 与湖泊研究所湖泊与环境国家重点实验室测试完成.

${ }^{210} \mathrm{~Pb}$ 和 ${ }^{137} \mathrm{Cs}$ 测定采用美国 EG \& GOrtec 公司生产的高纯锗井型探测器 (HPGe GwL-120-15) 进行, 用 Constant Rate of Supply (CRS) 模式建立钻孔沉积年代序列, 由于采用 CRS 模式计算得到的 1963 年的深度 与 ${ }^{137} \mathrm{Cs}$ 峰值不能对应, 故对该钻孔同样采用混合模式方法 (用 ${ }^{137} \mathrm{Cs}$ 时标对 CRS 模式进行校正, 固定 ${ }^{137} \mathrm{Cs}$ 的 峰值深度为 1963 年, 对 ${ }^{210} \mathrm{~Pb}$ 测年结果进行校正), 建立深度-年代关系 ${ }^{[21-22]}$.

按国际标准规范, 沉积物氮同位素采用 Deltaplus 质谱仪进行测定 ${ }^{[23]}$; 沉积物磷含量采用美国 LEEMAN LABS PROFILE 型电感耦合等离子体原子发射光谱仪 (ICP-AES) 测定 ${ }^{[23]}$; 金属元素含量采用全谱直读等离 子体发射光谱仪 (ICP-MS) 测定, 沉积物中重金属 Hg 含量采用直接永分析仪 (CVAAS 方法) 利用美国利曼 Hydra-C 全自动录分析仪测定.

硅藻样品依据标准方法 ${ }^{[24]}$ 进行处理. 取约 $0.2 \mathrm{~g}$ 沉积物, 先用 $10 \%$ 盐酸去钙质胶结物, 再用双氧水去有 机质, 离心清洗制片. 硅藻种属鉴定参照 Krammer 等的分类系统 ${ }^{[25]}$. 依据硅藻保存情况, 每个样品的硅藻统 计数控制在 300 粒以上,属种丰度用百分含量表示.

枝角类样品处理方法根据 Szeroczyńska 等 ${ }^{[26]}$ 的描述. 称取 3 5 g 样品加人 $100 \mathrm{ml} 10 \%$ 的 KOH 溶液浸 泡并在 $60^{\circ} \mathrm{C}$ 电热板上加热 $1 \mathrm{~h}$, 以去除有机质. 样品清洗后, 用 $38 \mu \mathrm{m}$ 的滤网进行过滤, 然后将滤网上的残留 物收集到离心管中并在 $4^{\circ} \mathrm{C}$ 冰箱中冷藏 $12 \mathrm{~h}$, 吸取上清液后加人 1 滴甲醛固定, 加人 $1 \sim 2$ 滴番红试剂进行染 色并定容到 $2 \mathrm{ml}$. 提取定量的处理好的样品, 在光学显微镜 (放大 200 倍)下进行种类鉴定. 每个样品中的统 
计数都达到 200 个以上, 以减小数据统计的误差. 统计时把所有化石残体 (头壳、壳瓣、后腹部、尾爪、卵鞍) 都进行统计, 选择数量最丰富的片断进行计数来代表枝角类个体数. 枝角类化石的分类鉴定主要参考 Szeroczyńska 等 ${ }^{[26]}$ 和 Jiang 等 ${ }^{[27]}$ 的图谱进行. 统计出的枝角类用相对丰度和沉积通量两种方式表达. 相对丰 度为样品中某个种的个体数占枝角类总个体数的百分比 (\%), 绝对丰度指单位重量沉积物中所含的枝角类 个体数 (ind. $/ \mathrm{g}(\mathrm{dw}))$.

\section{3 区域数据收集}

研究区最近 $60 \mathrm{a}$ 来的温度变化数据取自理塘县 (海拔 $3949 \mathrm{~m}$ ) 气象器测站 (来源于国家气候中心 http://ncc.cma.gov.cn/cn/). 根据小金县 1952 年以来的气象数据, 利用 ECOH-G 千年长积分模型重建了过 去 1000 年的年均温度变化 (闵㞨, 未发表数据). 截取近 200 年来的温度数据作为本研究的历史气候数据. 区域大气氮沉降数据采用喜玛拉雅山冰芯记录提取的硝酸盐 $\left(\mathrm{NO}_{3}^{-}\right)$浓度变化序列 ${ }^{[28]}$.

\section{4 数据分析}

根据枝角类数据的降维对应分析结果判识, 选择主成分分析 (PCA) 和尣余分析 (RDA) 来提取钻孔枝角 类群落变化的动态信息. 其中 PCA 分析 (只有枝角类相对丰度数据) 用于测试枝角类生态变化趋势; RDA 分 析 (同时包括枝角类丰度数据和环境指标数据) 用于测试环境因子与枝角类组合之间的关系, 采用蒙特卡罗 置换检验每个有效环境变量的显著性 ${ }^{[29]}$. RDA 分析中, 以枝角类数据作为响应变量, 环境指标 $(\mathrm{TN}$ 含量、 TOC 含量、 $\mathrm{C} / \mathrm{N}$ 比值、 $\delta^{15} \mathrm{~N}$, TP 含量和温度数据) 和作为解释变量. 在上述排序分析中选择至少在两个样品 中出现、至少在一个样品中含量超过 $1 \%$ 的属种 (共计 12 个). 所有排序分析均在 CANOCO 4.5 软件中 运行 ${ }^{[30]}$.

\section{3 研究结果}

\section{1 钻孔年代}

吉仁错沉积钻孔中 ${ }^{137} \mathrm{Cs}$ 比活度峰值 $(123.5 \pm 18.7 \mathrm{~Bq} / \mathrm{kg}$ ) 出现在 $12 \mathrm{~cm}$ (图 2a), 对应于 1963 年的散落高 峰期 ${ }^{[31]} \cdot{ }^{210} \mathrm{~Pb}_{\mathrm{ex}}$ 比活度从 $24 \mathrm{~cm}$ 开始增加, $11 \mathrm{~cm}$ 出现突然快速下降后, $10 \mathrm{~cm}$ 以上进一步增加. 由于采用 CRS 模式计算得到的 1963 年的深度与 ${ }^{137} \mathrm{Cs}$ 峰值不能对应, 故对该钻孔采用混合模式, 即用 ${ }^{137} \mathrm{Cs}$ 时标对 CRS 模式进行校正以建立深度-年代关系 ${ }^{[21-22]}$. 由于测定的 ${ }^{210} \mathrm{~Pb}_{\mathrm{ex}}$ 活度在 $21 \mathrm{~cm}$ 以下较低, 根据 $18 \sim 22.5 \mathrm{~cm}$ 段的 平均沉积速率来推算 $21 \mathrm{~cm}$ 深度以下的沉积物年代. 根据沉积物干通量 (DMAR) 计算, 沉积岩芯的平均沉 积速率为 $0.031 \mathrm{~g} /\left(\mathrm{cm}^{2} \cdot \mathrm{a}\right), 1900 \mathrm{AD}$ 之前 $(18 \sim 22.5 \mathrm{~cm})$ 沉积通量很低, 约为 $0.010 \mathrm{~g} /\left(\mathrm{cm}^{2} \cdot \mathrm{a}\right)$, 之后沉积 通量明显上升, 约为 $0.035 \mathrm{~g} /\left(\mathrm{cm}^{2} \cdot \mathrm{a}\right)$ ( 图 $2 \mathrm{~b}$ ).
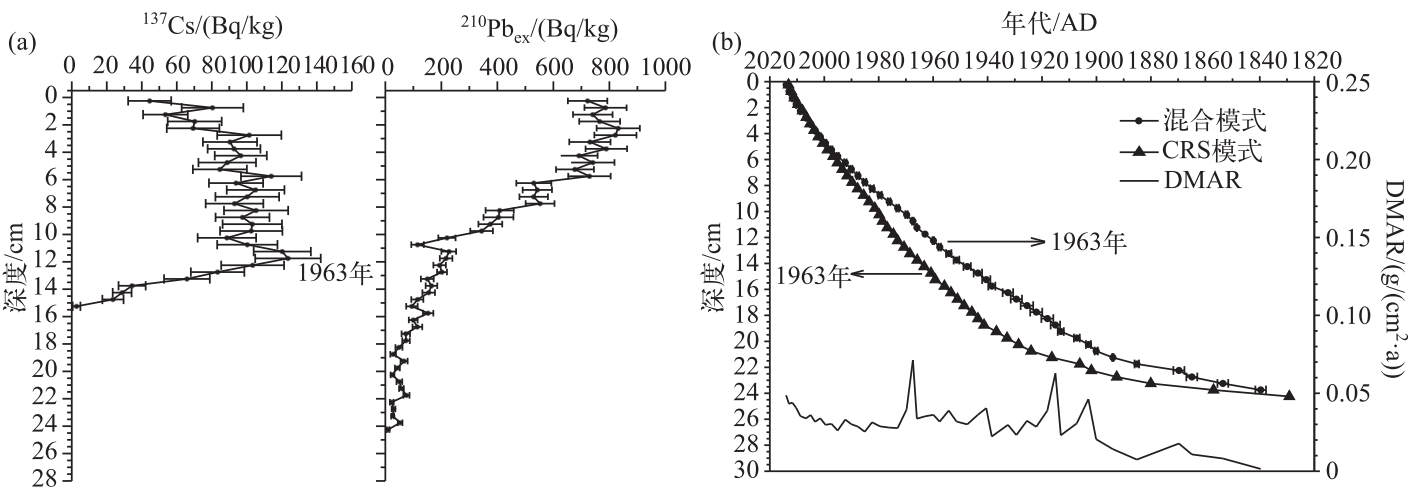

图 2 吉仁错钻孔 ${ }^{137} \mathrm{Cs}$ 和 ${ }^{210} \mathrm{~Pb}_{\mathrm{ex}}$ 比活度变化 (a), 及深度-年代序列和干沉积通量 (b)

Fig.2 The variation of ${ }^{137} \mathrm{Cs}$ and ${ }^{210} \mathrm{~Pb}_{\mathrm{ex}}$ activities from the sediment core in Jiren Co (a), as well as the age-depth model with dry mass accumulation rates (DMAR) (b) 


\section{2 枝角类化石沉积记录}

钻孔沉积物中共鉴定枝角类 12 个种, 隶属于 7 属, 优势种是沿岸种 (总含量 $>98 \%$ ), 包括 Alona guttata、 Alona rectangular、Alona affinis、Alona costata、Alonella nana、Acroperus harpae、Chydorus sphaericus, 其次是 Alona rustica、Alona quadrangularis、Pleuroxus trigonellus. 浮游种仅有少量记录,包括 Daphnia longispina 和 Bosmina sp.. 整个钻孔序列中, 枝角类组合以 P. trigonellus、C. sphaericus 和A. costata相对丰度增加, 以 A. rustica 和 A. nana 相对丰度显著下降为主要特征. 依据属种相对丰度数据的聚类分析结果 (图 3 ), 可划分为 3 个组 合带:

组合带 I( 28.5 24 cm, 1800-1850 AD) 以相对丰度较高的 A. guttata、A. rustica、A. harpae, 以及相对丰度 较低的 A. costata、C. sphaericus、P. trigonellus 为特征. A. affinis 和 A. rectangula 尽管也是本带的优势种,但在 整个剖面上含量保持相对稳定, 变化并不明显. 浮游种 D. longispina 和 Bosmina sp. 在本带偶见, 丰度极低. 枝 角类相对丰度数据的 PCA1 得分很低.

组合带 II (24 $14 \mathrm{~cm}, 1850-1945$ AD) 中 A. nana 相对丰度增加并达到剖面峰值 (最大值 20.7\%)， P. trigonellus相对丰度自本带开始缓慢增加, PCA1 得分波动增加. 相反, A. guttata、A. rustica 和 A. harpae 相 对丰度明显下降. 本带浮游种相对丰度略有升高, 主要是 D. longispina 的微弱增加.

组合带 III (14 cm 以上, 1945 AD 以来) 中,枝角类相对丰度数据的 PCA1 得分增加, 与 C. sphaericus 和 P. trigonellus相对丰度的持续增多相对应. A. rustica、A. nana 和 A. guttata 相对丰度快速降低. 本带浮游种 Bosmina sp. 和 Daphnia longispina 相对丰度有微弱增多.

钻孔中枝角类沉积通量整体呈上升趋势. $21 \mathrm{~cm}$ (1900 AD) 之前, 枝角类总通量很低 (16 51 ind./ $\left.\left(\mathrm{cm}^{2} \cdot \mathrm{a}\right)\right)$, 之后开始明显上升, 明显滞后于其相对丰度的变化. $14 \mathrm{~cm}(1945 \mathrm{AD})$ 以上枝角类通量再次上升 (平均为 $\left.111 \mathrm{ind} . /\left(\mathrm{cm}^{2} \cdot \mathrm{a}\right)\right)$, 与相对丰度变化一致.

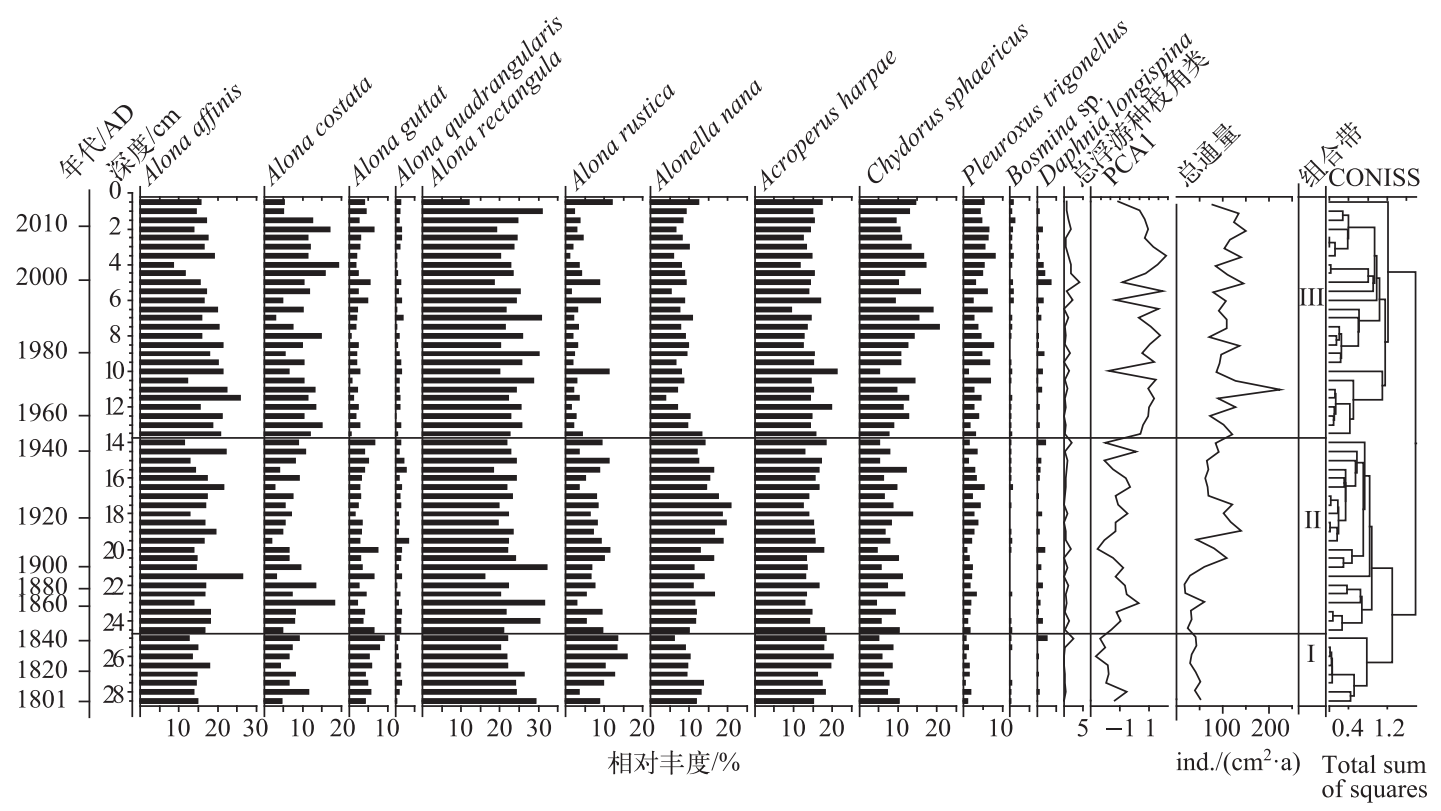

图 3 吉仁错钻孔中主要的化石枝角类属种组合以及枝角类总通量变化

Fig. 3 The diagram of cladoceran assemblage composition and total cladoceran flux from Jiren Co

\section{3 其他沉积指标与区域气候环境指标}

钻孔沉积硅藻优势种为浮游种 Aulacoseira pfaffiana、Cyclotella ocellata 以及小型的 Fragilaria 类型, 后者 又以 Fragilara construens var. venter 为主. 总体上, C. ocellata 与小型的 Fragilaria 类型的含量呈现互为消长的 关系. $1850 \mathrm{AD}$ 前, A. pfaffiana 含量为剖面峰值段, 此后呈逐渐下降趋势, C. ocellata 含量变化与之类似; 自 
1945 AD 以来,小型的 Fragilaria 的增多和 C. ocellata 的降低趋势更为明显. 硅藻总通量在 1900 AD 开始明 显上升,此后维持在高值阶段,与枝角类含量变化对应 (图 4).

沉积钻孔中各地球化学指标均在 $1900 \mathrm{AD}$ 前后出现微弱变化, $\delta^{15} \mathrm{~N}$ 值、 $\mathrm{C} / \mathrm{N}$ 比值和金属元素 $\mathrm{Ti}$ 含量开 始下降, 而 TOC 含量和重金属 Hg 含量略有升高. 明显的变化出现在 $1945 \mathrm{AD}$ 后, 与枝角类组合带 II 和组合 带 III 的界限一致. 剖面中沉积物磷含量在 1970s 后呈快速增高趋势 (图 4).

近 $60 \mathrm{a}$ 的温度监测记录显示 (图 4), 理塘县气温呈波动上升趋势, 尤其是 1990 年之后几乎呈直线上 升. 重建的区域温度变化显示, $1810 \mathrm{~s}-1850 \mathrm{~s}$ 年均温明显偏低, $1850 \mathrm{~s}$ 之后温度开始明显上升, 进人 20 世纪 温度总体呈波动上升趋势. 喜玛拉雅冰芯中 $\mathrm{NO}_{3}^{-}$浓度自 $1880 \mathrm{~s}$ 以来呈上升趋势, $1950 \mathrm{~s}$ 后进一步上升 ${ }^{[28]}$.

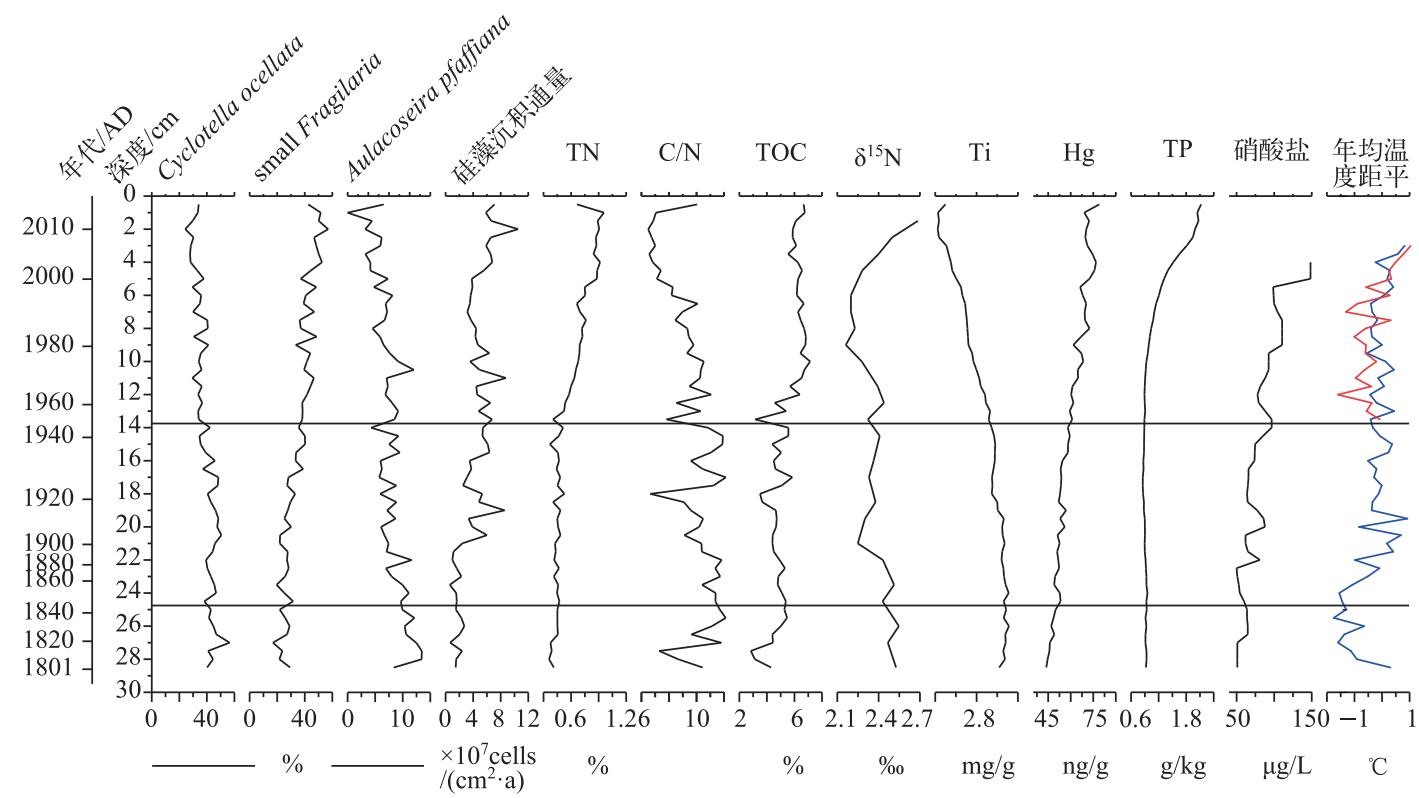

图 4 硅藻优势种、硅藻总通量、地球化学指标、喜马拉雅冰芯中的 $\mathrm{NO}_{3}^{-}$浓度以及

区域重建的温度 (蓝线) 和监测的理墉县 1952 年以来的温度 (红线))

Fig.4 The major diatom taxa, diatom flux, geochemistry proxies, $\mathrm{NO}_{3}^{-}$concentration, reconstructed annual temperature from Xiaojin county (blue line) and the monitor data from Litang county ( red line)

\section{4 枝角类和环境数据的 RDA 分析}

RDA 结果显示第 1 轴 (0.26) 和第 2 轴 (0.04) 解释 了枝角类数据中 $30.7 \%$ 的环境信息量 (图 5 ). 进一步选 择显示 $\mathrm{Ti}$ 含量、 $\mathrm{T}_{\text {winter }}$ (冬季温度) 和 $\mathrm{TN}$ 含量能显著解释 枝角类组合的变化 $(P<0.05)$. 从图 5 可以看出, 1945 $\mathrm{AD}$ 前的样点排列在第 1 轴负方向, 与 $\mathrm{Ti}$ 含量的增加相 对应, 其中早期 (1850 AD 以前) 样点处于第 1 轴的下方 向和冬季温度变量增加的反方向. $1945 \mathrm{AD}$ 以来的样品 主要分布在第 1 轴的正方向, 与冬季温度和 TN 含量的 增加相对应. 枝角类丰度的样点排列与组合带的划分 吻合.

\section{4 讨论}

吉仁错沉积枝角类记录揭示了近 $200 \mathrm{a}$ 来湖泊生态

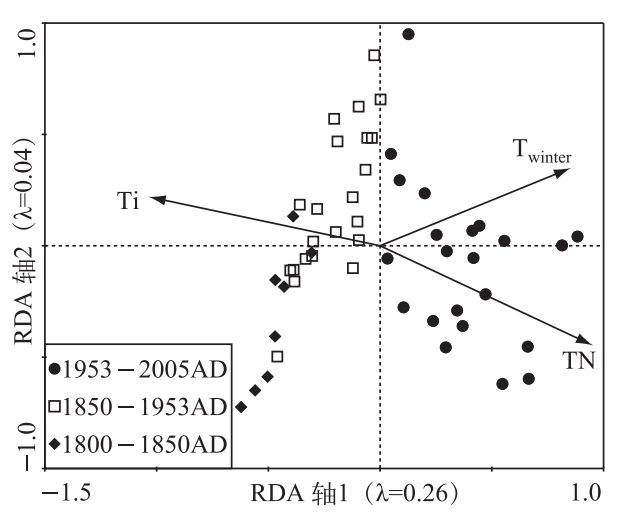

图 5 几余分析双轴图

Fig.5 The biplot of redundancy analysis 
环境的变化特征. 钻孔枝角类组合一直以沿岸的 Alona、Acroperus 和兼浮游种 Chydorus 种类组成为主, 浮游 种不发育. 根据枝角类个体生态学的特点, 这些沿岸优势种在高纬地区湖泊和温带高山湖泊中常见, 为典型 耐冷水种类 ${ }^{[17,20]}$, 说明过去 $200 \mathrm{a}$ 来湖泊水体温度总体很低. $1850 \mathrm{AD}$ 前, 高含量的极地种 A. harpae 和极低 的枝角类通量, 反映当时生物量很低,水温和营养水平极低; 而高含量的 A. guttata 和 A. rustica 的出现则指 示了相对较低的水体 $\mathrm{pH}$ 值 ${ }^{[32-33]}$, 与同时期硅藻组合中偏酸性的小型直链藻 A. pfaffiana 峰值段对应. 1850 $\mathrm{AD}$ 后, 逐渐增多的亚极地和温带种 P. trigonellus 以及偏酸性物种的下降表明当时水温和 $\mathrm{pH}$ 值开始缓慢升 高. 然而, 与含量变化不同的是, 钻孔枝角类通量的增高出现在 $1900 \mathrm{AD}$ 后, 与硅藻通量的增高时间相一致. 事实上, 营养的增加是湖泊生产力上升的首要前提 ${ }^{[6,34]}$, 推断 $1900 \mathrm{AD}$ 后枝角类生物量的增加可能与较高 的水体营养和食物来源有关. $1945 \mathrm{AD}$ 开始,亚极地种 A. costata 和兼浮游种 C. sphaericus 的显著上升、浮游 种类 Bosmina sp. 和 Daphnia longispina 的频繁出现、喜温水环境的 P. trigonellus 的持续增加以及持续的高枝 角类通量, 表明湖泊水温又有所上升, 生物量持续增高, 水体营养进一步提高. 此阶段喜好偏酸性环境的 A. guttata和 A. rustica 相对丰度持续下降, 暗示水体 $\mathrm{pH}$ 值较前期又有所上升, 与碱性底栖小型硅藻 Fragilaria 种类的持续增加趋势一致. 由此可见,湖泊水温、水体营养和 $\mathrm{pH}$ 值的变化, 是影响吉仁错枝角类 群落结构变化的关键环境过程.

钻孔多指标的对比、及其与区域气候和大气氮沉降记录的对比, 进一步揭示了增温和大气污染对湖泊 生态环境影响的证据. 钻孔剖面中, $1850 \mathrm{AD}$ 后枝角类组合变化指示的水温开始增加时间, 与小冰期结束区 域增温的时间相对应. 枝角类属种含量数据的 PCA 第 1 轴得分 (PCA1, 图 3) 与温度的相关分析也显示其生 态过程与冬季温度和年均温度都有较显著的相关性 (分别为 $r=0.54, P<0.05 ; r=0.66, P<0.05$ ). RDA 分析 显示冬季增温对该时间前后枝角类的显著影响关系. 推测在吉仁错, $1850 \mathrm{AD}$ 后增温不仅直接导致水体温度 的上升, 而且通过生长季节的加长, 对枝角类组合产生影响. $1890 \mathrm{AD}$ 后枝角类通量的开始增加、以及 1945 $\mathrm{AD}$ 后通量的进一步增加, 与环境指标 $\mathrm{Ti}$ 含量、 $\delta^{15} \mathrm{~N}$ 值、C/N 比值的逐渐降低和 $\mathrm{Hg}$ 含量的持续增高相对应. $\mathrm{Hu}$ 等 ${ }^{[6]}$ 认为研究区高山湖泊钻孔中 $\mathrm{Ti}$ 含量的下降指示了流域植被的发育, 而流域植被的发育一方面可能 与增温有关, 另一方面也与该流域营养补充有关. 在无人类直接干扰的高山湖泊, $\delta^{15} \mathrm{~N}$ 值的下降通常指示大 气氮沉降的影响 ${ }^{[35]}, \mathrm{Hg}$ 含量的增加则与大气污染有关 ${ }^{[36]}$, 两者变化与来自喜马拉雅冰芯的 $\mathrm{NO}_{3}^{-}$浓度变化 的一致性,进一步表明了近百年来大气氮沉降对湖泊生态的影响. $1890 \mathrm{AD}$ 以来 $\mathrm{C} / \mathrm{N}$ 比值的持续降低可能 反映该湖初级生产力显著增加的过程, 并得到钻孔硅藻通量增加的相互印证. 钻孔顶部枝角类生物量达到 最大, 与最近几十年来 TP 浓度的快速升高对应. 有研究表明, 在无人类直接影响的地区, 进人到水体中的磷 主要来自大气粉尘直接沉降 ${ }^{[37]}$, 青藏高原东南缘地区超过 $80 \%$ 的总磷 $\left(50 \sim 100 \mathrm{~g} /\left(\mathrm{hm}^{2} \cdot \mathrm{a}\right)\right)$ 来源于尘 降 $^{[38]}$. 由此可见, 过去 $100 \mathrm{a}$ 来区域大气氮沉降以及近 30 多年来粉尘污染的加强, 应该是吉仁错水体营养 持续增加的主要原因, 并进而导致藻类食物来源增多, 促进了枝角类生物量的提高.

然而, 尽管枝角类组合反映了过去 $150 \mathrm{a}$ 来湖泊水温缓慢增高的过程, 但大量偏好冷水环境的沿岸种的 维持暗示水温增加有限, 浮游种的发育仍受到限制. 而且无论是温度还是营养变化, 都无法解释由枝角类和 硅藻反映的水体 $\mathrm{pH}$ 值的明显增加. 此外, 钻孔剖面中 $\mathrm{Ti}$ 含量的下降反映了流域稳定性自 20 世纪初期以来 缓慢增强的趋势, 但 RDA 分析揭示了 Ti 含量和 TN 含量呈明显负相关, 且共同显著影响了枝角类群落组合 的变化, 暗示流域植被和土壤过程, 通过营养输人, 在一定程度上对湖泊枝角类群落结构产生影响. 我们认 为,气候和大气氮沉降不仅对湖泊生态环境产生直接影响, 而且间接地通过流域冰川积雪融水和植被土壤 过程, 对湖泊生态系统产生影响. 吉仁错流域目前仍有小面积山地冰川发育, 湖泊受到冰川和周围积雪融水 的补给. $1850 \mathrm{AD}$ 后水体 $\mathrm{pH}$ 值的上升与增温引起的流域冰川积雪融水补给量的增多有关, 而后者增加了湖 泊碱性溶解物质的补给, 进而影响水体酸碱平衡 ${ }^{[39]}$. Anderson 等 ${ }^{[29]}$ 研究发现, 在边远地区湖泊, 温度上升会 加快流域风化速率和碱性物质生成, 通过冰融水等径流, 导致湖泊碱化进而明显地影响 $\mathrm{pH}$ 值. 在吉仁错, 流 域冰雪融水注人的增多还会部分抵消或减缓由于增温引起的表层水温的增加, 进而限制浮游枝角类生长. Slemmons 等 ${ }^{[40]}$ 通过对比研究认为阿拉斯加山地冰川融水补给的湖泊, 平均水温甚至较无冰川融水补给的 湖泊还要低 $2^{\circ} \mathrm{C}$ 以上. 不难理解过去 $150 \mathrm{a}$ 来该湖水体温度尽管有所上升, 枝角类组合仍以极地和亚极地沿 岸种类占优势地位. 
已有的研究发现,冰川融水补给的湖泊比其他仅有降水和积雪补给的湖泊更易富集氮, 尤其是 $\mathrm{NO}_{3}^{-[41]}$. 冰川作为高海拔地区大气化学物质沉降的主要积蓄库, 冰融水的增加也促进了人湖活性氮、溶解有机物、重 金属含量和其他营养物质如总磷含量的增加 ${ }^{[42-44]}$. 吉仁错钻孔沉积物中, $1945 \mathrm{AD}$ 后 TN 含量的明显上升, 应该是气候持续增暖情况下, 通过流域冰雪融水过程的加强, 输人的大气 $\mathrm{N}$ 沉降导致的, 并部分受到流域植 被和土壤微生物的作用. 与此同时, 流域冰雪融水使得碱性物质输人量持续增多, 导致湖水 $\mathrm{pH}$ 值的进一步 升高. 推测 $1945 \mathrm{AD}$ 前后吉仁错枝角类和藻类群落结构和湖泊环境的同步变化, 共同响应于气候、大气沉 降、流域冰雪融水和风化过程、植被和土壤发育过程的相互作用.

\section{5 结论}

1 ) 吉仁错钻孔枝角类记录揭示了过去 $200 \mathrm{a}$ 来一直以沿岸种为优势组合的特点, 并经历了由 A. guttata、 A. rustica 和 A. harpae 为主 (1850 AD 前)、过渡到以 A. nana 和 P. trigonellus 增多(1850-1945 AD 期间) 和 A. costata、C. sphaericus 和 P. trigonellus 为优势 (1945 AD 以来) 的 3 个变化阶段. 枝角类生物量经历了由低 (1900 AD 前) 到较高 (1900-1945 AD) 再到高 (1945 AD 以来) 的变化过程. 枝角类群落结构变化揭示了湖 泊水温、水体营养和 $\mathrm{pH}$ 值变化的环境信息.

2)过去 $150 \mathrm{a}$ 来,气候变暖和大气污染沉降通过直接和间接作用(流域冰川融水过程和植被土壤过 程), 较深刻地影响了湖泊物理过程 (水温升高和无冰期加长) 、营养过程 (氮、磷营养升高) 和酸碱平衡过程 (碱性增强), 改变了湖泊生物的生长季节, 并通过促进藻类发育, 最终引起了湖泊枝角类群落组合的变化和 生物量的增加.

致谢: 英国拉夫堡大学 John Anderson 教授和中国科学院南京地理与湖泊研究所赵成研究员协助野外采样; 中国科学院南京地理与湖泊研究所夏威岗老师、朱育新老师、刘一兰老师分别提供了放射性测年结果、金属 元素、氮同位素及总有机碳结果, 胡竹君博士提供了钻孔硅藻数据. 在此一并感谢.

\section{6 参考文献}

[ 1 ] IPCC. Climate change 2013: The physical science basis. New York: Cambridge University Press, 2014.

[ 2 ] Beniston M, Diaz HF, Bradley RS. Climatic change at high elevation sites: An overview. Climatic Change, 1997, 36(3): 233-251.

[ 3 ] Galloway JN, Cowling EB. Reactive nitrogen and the world: 200 years of change. AMBIO: A Journal of the Human Environment, 2002, 31(2): 64-71.

[ 4 ] Smol JP. Pollution of lakes and rivers: A paleoenvironmental perspective. John Wiley \& Sons, 2009.

[ 5 ] Ørbæk JB, Kallenborn R, Tombre I et al. Arctic alpine ecosystems and people in a changing environment. Springer Science \& Business Media, 2007.

[ 6 ] Hu Z, Anderson NJ, Yang X et al. Catchment-mediated atmospheric nitrogen deposition drives ecological change in two alpine lakes in SE Tibet. Global Change Biology, 2014, 20(5) : 1614-1628.

[ 7 ] Liu X, Zhang Y, Han W et al. Enhanced nitrogen deposition over China. Nature, 2013, 494(7438): 459-462.

[ 8 ] Yan Ting, Liu Enfeng, Zhang Enlou et al. Atmospheric Pb pollution in the alpine area of southwest China based on the lichens (Usnea longissima) analysis. China Environmental Science, 2015, 35(9): 2772-2777. [燕婷, 刘恩峰, 张恩楼等. 基于地衣植物监测法的我国西南高山地区大气铅污染研究. 中国环境科学, 2015, 35(9): 2772-2777.]

[ 9 ] Battarbee RW, Morley D, Bennion H et al. A palaeolimnological meta-database for assessing the ecological status of lakes. Journal of Paleolimnology, 2011, 45(4) : 405-414.

[10] Holtmeier FK, Broll G. Treeline advance-Driving processes and adverse factors. Landscape Online, 2007, 1: 1-33.

[11] Hood EW, Williams MW, Caine N. Landscape controls on organic and inorganic nitrogen leaching across an alpine/subalpine ecotone, Green Lakes Valley, Colorado Front Range. Ecosystems, 2003, 6(1) : 31-45.

[12] Myers N, Mittermeier RA, Mittermeier CG et al. Biodiversity hotspots for conservation priorities. Nature, 2000,403 (6772) : 853-858.

[13] Wang Rong, Yang Xiangdong, Zhu Liping. Environmental changes of Namu Co, Xizang during the past 200 years. Quaternary Sciences, 2006, 26(5):791-798. [王荣, 羊向东, 朱立平. 西藏纳木错过去 200 年来的环境变化. 第四纪研究, 
2006, 26 ( 5 ): :791-798. ]

[14] Wischnewski J, Kramer A, Kong Z et al. Terrestrial and aquatic responses to climate change and human impact on the southeastern Tibetan Plateau during the past two centuries. Global Change Biology, 2011, 17(11) : 3376-3391.

[15] Wischnewski J, Mackay AW, Appleby PG et al. Modest diatom responses to regional warming on the southeast Tibetan Plateau during the last two centuries. Journal of Paleolimnology, 2011, 46(2) : 215-227.

[16] Kong L, Yang X, Kattel G et al. The response of Cladocerans to recent environmental forcing in an Alpine Lake on the SE Tibetan Plateau. Hydrobiologia, 2017, 784(1) : 171-185.

[17] Lotter AF, Birks HJB, Hofmann W et al. Modern diatom, cladocera, chironomid, and chrysophyte cyst assemblages as quantitative indicators for the reconstruction of past environmental conditions in the Alps. I. Climate. Journal of Paleolimnology, 1997, 18(4) : 395-420.

[18] Lotter AF, Birks HJB, Hofmann W et al. Modern diatom, cladocera, chironomid, and chrysophyte cyst assemblages as quantitative indicators for the reconstruction of past environmental conditions in the Alps. II. nutrients. Journal of Paleolimnology, 1998, 19(4): 443-463.

[19] Jeppesen E, Leacitt P, Meester LD et al. Functional ecology and palaeolimnology : Using cladoceran remains to reconstruct anthropogenic impact. TRENDS in Ecology \& Evolution, 2001, 16: 191-198.

[20] Bigler C, Heiri O, Krskova R et al. Distribution of diatoms, chironomids and cladocera in surface sediments of thirty mountain lakes in south-eastern Switzerland. Aquatic Sciences, 2006, 68(2) : 154-171.

[21] Appleby PG. Chronostratigraphic techniques in recent sediments. In: Last WM, Smol JP eds. Tracking environmental change using lake sediments. Volume 1: Basin analysis, coring and chronological techniques. Dordrecht: Kluwer Academic Publishers, 2001: 171-203.

[22] Liu Enfeng, Xue Bin, Yang Xiangdong et al. ${ }^{137} \mathrm{Cs}$ and ${ }^{210} \mathrm{~Pb}$ chronology for modern lake sediment: A case study of Chaohu lake and Taibai lake. Marine Geology \& Quaternary Geology, 2009, (6) : 89-94. [ 刘恩峰, 薛滨, 羊向东等. 基于 210 $\mathrm{Pb}$ 与 ${ }^{137} \mathrm{Cs}$ 分布的近代沉积物定年方法一一巢湖, 太白湖为例. 海洋地质与第四纪地质, 2009, (6) : 89-94.]

[23] Hu Zhujun. Ecological changes and corresponding mechanisms in alpine lakes of the southeast margin of Tibetan Plateau [Dissertation]. Nanjing: University of Chinese Academy of Science, 2013. [ 胡竹君. 青藏高原东南缘高山湖泊生态变 化与驱动机制 [学位论文]. 南京: 中国科学院南京地理与湖泊研究所, 2013.]

[24] Battarbee RW, Cameron NG, Golding P et al. Evidence for Holocene climate variability from the sediments of a Scottish remote mountain lake. Journal of Quaternary Science, 2001, 16(4) : 339-346.

[25] Krammer K, Lange-Bertalot H. Bacillariophyceae 1-4. In: Ettl H, Gerloff J, Heynig H et al eds. Süsswasserflora von Mitteleuropa, Band 1-4. Jena: Gustav Fischer Verlag, 1986-1991.

[26] Szeroczyńska K, Sarmaja-Korjonen K. Atlas of subfossil Cladocera from central and northern Europe. Swiecie: Friends of the Lower Vistula Society, 2007: 10-11.

[27] Jiang Xiezhi, Du Nanshan eds. Fauna Sinica Crustacer Freshwater Cladocera. Beijing: Science Press, 1979.[蒋泶治, 堵 南山. 中国动物志，节肢动物门，甲壳纲，淡水枝角类. 北京: 科学出版社，1979.]

[28] Thompson LG, Yao T, Mosley-Thompson E et al. A high-resolution millennial record of the South Asian monsoon from Himalayan ice cores. Science, 2000, 289: 1916-1919.

[29] Anderson NJ, Brodersen KP, Ryves DB et al. Climate versus in-lake processes as controls on the development of community structure in a low-arctic lake (South-West Greenland). Ecosystems, 2008, 11(2) : 307-324.

[30] Ter Braak CJF, Šmilauer P eds. Canoco for windows 4.5. Wageningen: Centre for Biometry, 2002.

[31] Wan Guojiang. Progresses on ${ }^{137} \mathrm{Cs}$ and ${ }^{210} \mathrm{~Pb}_{\mathrm{ex}}$ dating of lake sediments. Advances in Earth Science, 1995, (2) : 188-192. [万国江. ${ }^{137} \mathrm{Cs}$ 及 ${ }^{210} \mathrm{~Pb}_{\mathrm{ex}}$ 方法湖泊沉积计年研究新进展. 地球科学进展, 1995，(2): 188-192.]

[32] Walseng B, Halvorsen G, Sloreid SE. Littoral microcrustaceans (Cladocera and Copepoda) as indices of recovery of a limed water system. Hydrobiologia, 2001, 450(1/2/3): 159-172.

[33] Korosi JB, Smol JP. Examining patterns in the distribution of cladoceran assemblage and size structure in Nova Scotia Canada. Hydrobiologia, 2011, 663: 83-99.

[34] Bergström Annk, Jansson M. Atmospheric nitrogen deposition has caused nitrogen enrichment and eutrophication of lakes in the northern hemisphere. Global Change Biology, 2006, 12(4) : 635-643.

[35] Holtgrieve GW, Schindler DE, Hobbs WO et al. A coherent signature of anthropogenic nitrogen deposition to remote water- 
sheds of the northern hemisphere. Science, 2011, 334(6062) : 1545-1548.

[36] Kang Shichang, Huang Jie, Zhang Qianggong. Progress in the study of mercury in sonw and ice. Advances in Earth Science, 2010, 25(8): 783-793. [ 康世昌, 黄杰, 张强弓. 雪冰中求的研究进展. 地球科学进展, 2010, 25 ( 8): 783-793.]

[37] Kalff J ed. Limnology: Inland water ecosystems. New Jersey: Prentice Hall, 2002.

[38 ] Mahowald N, Jickells TD, Baker AR et al. Global distribution of atmospheric phosphorus sources, concentrations and deposition rates, and anthropogenic impacts. Global Biogeochemical Cycles, 2008, 22(4) : 37-42.

[39] Koinig KA, Schmidt R, Sommaruga-Wögrath S et al. Climate change as the primary cause for $\mathrm{pH}$ shifts in a high alpine lake. Water, Air, and Soil Pollution, 1998, 104(1/2): 167-180.

[40] Slemmons KEH, Saros JE, Simon K. The influence of glacial meltwater on alpine aquatic ecosystems: A review. Environmental Science: Processes \& Impacts, 2013, 15(10) : 1794-1806.

[41] Blais JM, Schindler DW, Muir DCG et al. Melting glaciers: A major source of persistent organochlorines to subalpine Bow Lake in Banff National Park, Canada. Ambio, 2001, 30: 410-415.

[42] Fowler D, Battarbee R. Climate change and pollution in the mountains: The nature of change. Mountains of Northern Europe: Conservation, management, people, and nature. Edinburgh: The Stationery Office, 2005: 71-88.

[43] Thies H, Nickus U, Mair V et al. Unexpected response of high alpine lake waters to climate warming. Environmental Science \& Technology, 2007, 41(21): 7424-7429.

[44] Saros JE, Rose KC, Clow DW et al. Melting alpine glaciers enrich high-elevation lakes with reactive nitrogen. Environmental Science \& Technology, 2010, 44(13): 4891-4896. 